Published in La Revue des Sciences de Gestion, 2016, vol.5 no.281-282,

p.151-161 which should be cited to refer to this work

La Revue des Sciences de Gestion, Direction et Gestion n 281-282 - organisation

\title{
Management et culture du risque de crédit dans les banques tunisiennes
}

par Hichem Maraghni, Faten Ben Bouheni, Mondher Bellalah, Lubica Hikkerova et Jean-Michel Sahut

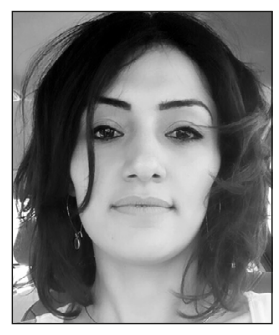

Faten BEN BOUHENI

Enseignant-Chercheur

ISC Paris Business School

Chercheur associé au LITEM, Paris

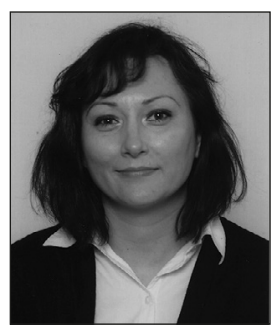

Lubica HIKKEROVA

Enseignant-Chercheur

IPAG Business School, Paris

France

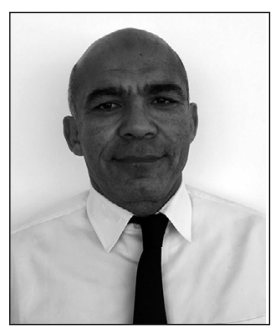

\author{
Mondher BELLALAH \\ Professeur \\ Université de Cergy-Pontoise \\ ISC Paris Business School, France
}

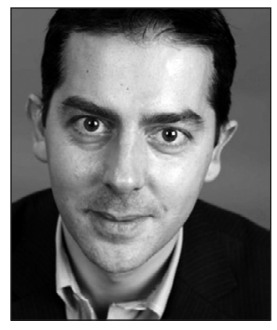

Jean-Michel SAHUT

Enseignant-Chercheur, IDRAC Business School ( $\mathrm{Fr}_{\mathrm{r}}$, HEG Fribourg, HES-SO/University of Applied Sciences Western Switzerland, Suisse 
(réglementation de Bâle I) a été à la source d'une culture limitée du risque dans les banques des pays développées (D. Ospital, 2006), d'où le renforcement des règles prudentielles avec les comités Bâle II puis Bâle III. De plus, la transposition de normes internationales dans des pays émergents, alors qu'elles ont été élaborées sous la direction de pays développés, risque de produire des effets différents (M. Arouri et al., 2013). En fait, les normes comme les valeurs de la société influencent la culture de toute entreprise, laquelle est une variable essentielle pour expliquer les pratiques et les choix stratégiques réalisés par un groupe social (B. Massiera, 2007).

Cet article vise donc à analyser la gestion et la culture du risque des banques dans le contexte d'un marché émergent, la Tunisie, suite à une évolution du cadre réglementaire dicté par la supervision bancaire. II analyse l'effet simultané entre les variations du ratio de capital réglementaire et le niveau de la prise de risque de crédit des banques universelles sous la pression réglementaire (M. Bellalah, 2006). Notre analyse empirique repose sur un modèle structurel en double équations simultanées pour la période de 1990 à 2012.Dans ce modèle, développé au départ dans les travaux de D. Dahl et R. Shrieves (1990), R. Aggarwal et K.T. Jacques (1998) et P. Van Roy (2005), nous avons introduit quelques transformations telles que celles réalisées récemment par T. Jokipii et A Milne (2010) et S.L. Lin et al. (2013), dans le but de les adapter au contexte tunisien.

Les contributions de notre article en matière de management du risque et de culture reposent sur l'originalité de notre démarche méthodologique et de notre échantillon. En effet, la plupart des travaux existants se sont focalisés sur les banques européennes ou américaines alors que notre article étudie la gestion et la culture du risque de crédit dans un pays émergent, la Tunisie où les grandes banques ont bien résisté à la récente crise financière de 2007-2008 (M. Arouri et al., 2013). Nos résultats apportent des éléments de réponse sur le comportement des banques tunisiennes et leur culture du risque. En effet, les normes de gestion prudentielle mises en place par les autorités réglementaires tunisiennes avaient pour objectif de limiter les prises de risque des banques (de crédit, de liquidité, de marché, opérationnel, etc.) et leurs engagements dans les activités les plus risquées, ainsi que de superviser l'activité bancaire par la vérification sur place et sur pièces du respect de ces exigences. Cette intervention d'ordre juridique s'est avérée indispensable pour assurer la solidité et la pérennité du système bancaire, mais des recherches complémentaires paraissent indispensables afin de mieux comprendre ses impacts sur la culture du risque des banques. L'effet est-il aussi limité que lors de l'introduction du "ratio Cooke"?

Pour répondre à cette problématique, nous rappellerons dans un premier temps, le cadre de la gestion du risque de crédit et des ratios prudentiels dans les banques, et développerons un modèle pour analyser le comportement des banques en termes de prise de risque de crédit par rapport au ratio de solvabilité. Dans un second temps, nous présenterons notre méthodologie, la construction de notre base de données et quelques statistiques descriptives de nos variables. Enfin, dans un troisième temps, nous exposerons et analyserons l'essentiel des résultats des estimations de la relation entre les variations du ratio du capital et les variations du niveau du risque de crédit, moyennant un modèle à deux équations simultanées. Bien sûr, nous nous intéresserons à chaque étape à la réaction du management et la culture du risque des banques.

\section{Gestion du risque et ratios prudentiels}

\subsection{Cadre d'analyse et hypothèses}

Pour les autorités réglementaires d'un pays, le problème est de définir les outils pour contrôler et superviser la santé financière des banques opérant sur son territoire. C'est ainsi qu'est né, dans les années 1970, le concept de ratio de solvabilité (M.C. Chiuri et al., 2002) ou Capital Adequacy Ratio (CAR). L'expansion du crédit par les banques sans une augmentation en parallèle de leur capital a contraint les organismes de réglementation à définir au fil du temps plusieurs procédures de contrôle et émettre de nouvelles réformes afin d'éviter l'insolvabilité du secteur bancaire en cas de crise. Dans ce contexte (à savoir, la protection contre les chocs), les exigences en matière de capital sont les instruments les plus fréquemment utilisés par la réglementation prudentielle. L'importance de cet instrument pour assurer la stabilité bancaire a incité de nombreux chercheurs à étudier ses déterminants et la manière dont il influence la prise de risque des banques. En particulier, J. Ayuso et al. (2004) et R.S. Demsetz et P.E. Strahan (1997) ont mis en évidence que les grandes banques peuvent fonctionner avec un ratio de capital réglementaire plus faible que les petites banques car elles bénéficient d'effet de diversification des risques. Par ailleurs, R. Gropp et F. Heider (2009) et V. Kleff et M. Weber (2008) ont affirmé que les banques les plus rentables ont tendance à avoir un ratio de capital réglementaire relativement plus élevé. Plus largement, d'autres auteurs comme P. Calem et R. Rob (1999) ont découvert que l'accroissement de ce ratio réglementaire a entraîné une hausse du niveau du capital des banques et une diminution limitée de la prise de risque. Sur le plan réglementaire, les établissements dont le portefeuille est jugé relativement risqué (avec un niveau de risque de crédit élevé), sont obligés de détenir un niveau de fonds propres (qui constitue un tampon) plus important car ils sont susceptibles de se trouver avec des ratios de capital minimum inférieurs à la norme réglementaire exigée. Cette situation est de nature à accroître leur probabilité de faillite, étant incapables de faire face aux coûts associés à cette situation financière délicate. Sur la base des arguments précédents, nous nous proposons de tester les deux hypothèses suivantes:

- Hypothèse 1 (H1) : La variation du niveau du ratio de capital de la banque a un effet significativement négatif sur le comportement de prise de risque de crédit. 
- Hypothèse 2 (H2) : La variation du niveau de la prise de risque de crédit est corrélée négativement avec le changement du niveau du ratio du capital.

Récemment, T. Jokipii et A. Milne (2010), S.L. Lin et al. (2013); en étudiant la relation entre la réserve de capital (Capital Buffer), le capital, le risque, la performance et le rendement; ont identifié un comportement contra-cyclique de cette réserve, particulièrement sur le niveau du risque de crédit en Suisse, et aux Etats-Unis. En se référant à la théorie couramment appelée "Buffer Theory", on s'attend à ce que les ajustements du niveau de fonds propres avec celui de la prise de risque de crédit soient positivement corrélés si les fonds propres tampon ou de sécurité sont relativement élevés, tandis qu'ils seront négativement liés sinon. Selon cette approche, les banques, qui affichent des ratios de solvabilité supérieurs à la norme réglementaire exigée (elles sont alors suffisamment capitalisées), possèdent des fonds réserve de capital plus confortables.

Toutefois, pour les banques qui s'approchent du niveau requis de capital réglementaire, la relation pourrait être négative à court terme. En effet toute augmentation des exigences de fonds propres réglementaires $(\triangle \mathrm{CAR})$ se traduit par une baisse du niveau du stock de réserve en capital (BUFFER) et, inversement, une hausse du niveau des fonds propres tampon se traduit par une dégradation du ratio de capital. Ainsi, au vu des informations sur les niveaux relativement faibles des valeurs de (BUFFER) et ( $\triangle \mathrm{CAR}$ ) pour les banques tunisiennes (Rapport de la $B C T^{1}, 2013$ ), nous formulons notre troisième hypothèse de la façon suivante :

- Hypothèse 3 (H3) : Le niveau des fonds propres tamponest associé négativement aux variations du niveau du capital.

\subsection{Spécification économétrique du modèle et ajustement partiel}

Dès 1968, E.I. Altman a souligné qu'en plus de leur utilité traditionnelle d'évaluation des performances financières et économiques des établissements de crédit, les ratios financiers peuvent être considérés comme des outils de rationalisation interne et statique, nécessaires pour la prédiction des difficultés. B. Lev (1969) a ensuite montré que le ratio moyen d'un secteur d'activité est souvent utilisé comme valeur "cible" par les firmes pour s'ajuster, et en a déduit un modèle d'ajustement partiel, qui a été ensuite amélioré par les travaux de J. Lintner (1977) dans le cadre de l'ajustement du niveau cible des dividendes des firmes bancaires. C'est dans le cadre de ces travaux d'ajustement partiel que s'inscrit notre contribution à travers un modèle à deux équations simultanées développé initialement par D. Dahl et R. Shrieves (1990) et repris par K.T. Jacques et P. Nigro (1997), T. Ediz et al. (1998), Z. Saadaoui (2010) et S.L. Lin et al. (2013), afin d'étudier la relation entre le niveau du ratio de capitalisation et le niveau du ratio de risque des firmes bancaires.
La revue de la littérature financière a d'ailleurs montré que ces deux variables endogènes sont reliées et se comportent d'une façon simultanée (R. Aggarwal et K.T. Jacques, 1998). En d'autres termes, deux situations possibles se présentent: a) Chaque variation du niveau du ratio de capitalisation peut entraîner un ajustement du niveau d'incitation à la prise de risque de crédit, et b) Toute éventuelle évolution du niveau du risque de crédit est susceptible d'inciter l'établissement à ajuster son ratio de capitalisation vers une valeur cible souhaitée pour se couvrir contre ce risque.

Les valeurs cibles du ratio de capital (CAR*) ainsi que celle du niveau du risque de crédit (CRRISK*), que les établissements de crédit agréés en qualité de banque désirent obtenir dépendent de plusieurs variables exogènes liées au cadre institutionnel dans lequel elles évoluent. À long terme, ces banques souhaitent atteindre des degrés optimaux de ratios suivant un ajustement partiel. Ainsi, D. Dahl et R. Shrieves (1990) ont précisé que ces firmes sont incapables d'atteindre instantanément les niveaux "ciblés" et le font de façon partielle, d'où l'utilisation des paramètres $(\lambda)$ et $(\gamma)$ liés à cet ajustement (sachant que leurs valeurs varient entre zéro et un). Au cours de chaque année (t) et pour chaque banque (i), le changement optimal du niveau du ratio du capital et du niveau de ratio du risque de crédit sera motivé par la différence entre CAR* et CAR $t-1^{2}$ et simultanément entre CRRISK* et CRRISK $t-1$.

Ce qui se traduit dans les deux équations (I.1) et (I.2) suivantes par :

$\Delta$ CAR $_{\mathrm{i}, \mathrm{t}}=\lambda\left(\mathrm{CAR}^{*}-\mathrm{CAR}_{\mathrm{t}-1}\right)$

$\Delta$ CRRISK $_{\mathrm{i}, \mathrm{t}}=\gamma\left(\right.$ CRRISK $^{\star}-$ CRRISK $\left._{\mathrm{t}-1}\right)$

En tenant compte des variables explicatives (exogènes) telles qu'elles sont mentionnées dans les travaux de K.T. Jacques et P. Nigro (1997)etS.L. Lin et al. (2013), dans les deux équations simultanées, respectivement $\left(E_{t}\right)$ et $\left(S_{t}\right)$; nous obtenons les spécifications économétriques de base (I.3) et (I.4) qui s'énoncent mathématiquement comme suit :

$\Delta$ CAR $_{\mathrm{i}, \mathrm{t}}=\lambda\left(\mathrm{CAR}^{\star}-\mathrm{CAR}_{\mathrm{t}-1}\right)+\mathrm{Et}$

$\Delta$ CRRISK $_{\mathrm{i}, \mathrm{t}}=\gamma\left(\right.$ CRRISK $^{\star}-$ CRRISK $\left._{\mathrm{t}-1}\right)+\mathbf{S t}$

Notre modèle estimé s'écrit sous la forme des deux équations simultanées (I.5.1) et (I.5.2) dans le système suivant (I.5) :

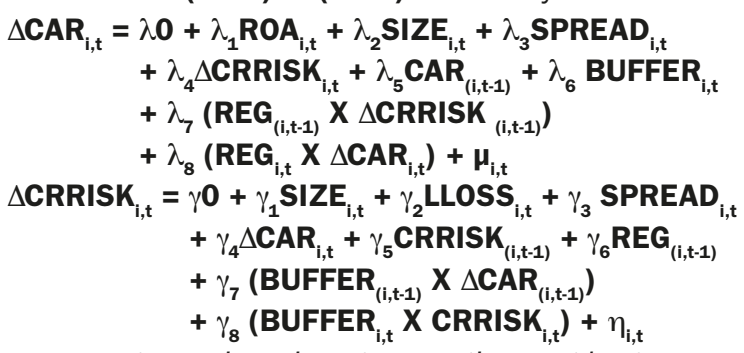

avec, $\mu_{i, t}$ et $\eta_{i, t}$ qui expriment respectivement les termes de résidus des équations de niveau de capitalisation et du niveau du risque de crédit pour l'établissement (i) au cours de l'exercice (t). Nous notons également que $\lambda j(j=1 \ldots 8)$ et $\gamma k(k=1 \ldots 8)$, sont les paramètres du modèle affectés aux différentes variables et

2. Valeurs considérées durant l'exercice antérieur (t-1) 
( $\lambda 0)$ et $(\gamma 0)$ sont les constantes respectives aux deux équations simultanées du modèle (I.5).

Les deux équations (1.5.1) et (1.5.2), qui forment notre modèle, expriment simultanément la relation entre le niveau de variation du capital et le comportement des banques en termes de prise de risque de crédit (deux variables endogènes). Ces dernières sont fonction des niveaux cibles souhaités des fonds propres et de risque de crédit ${ }^{3}$ ainsi qu'une batterie de facteurs explicatifs appropriés (variables exogènes) que nous présentons ci-dessous puis dans le tableau 2.

CAR : c'est le ratio de solvabilité ou d'adéquation des fonds propres réglementaires, appelé aussi ratio "McDonough ". Il est défini comme le rapport entre les fonds propres nets prudentiels de base (Tier 1) et complémentaires (Tier 2), et les actifs bancaires pondérés ou ajustés en fonction des différentes classes de risque $^{4}$. Ce ratio, ne pouvant pas être calculé directement à partir des états financiers, il est généralement publié par les banques dans leurs rapports annuels sous la forme de la variation première entre deux exercices $\triangle C A R i t=C A R i, t-C A R i, t-1$ Le ratio (CAR) qui mesure la solvabilité de la banque, est devenue très populaire, comme l'atteste A. Powell (2004), depuis son adoption par les autorités réglementaires internationales. La valeur de ce ratio doit être supérieure à $8 \%$ depuis le début de l'année 2000 en Tunisie (contre $5 \%$ auparavant). II a été utilisé par K.T. Jacques et P. Nigro (1997), F. Heid et al. (2004), T. Jokipii et A. Milne (2010) et S.L. Lin et al. (2013) pour cerner l'effet simultané entre capital et risque de contrepartie, mesuré par le ratio (RWA/TA) ${ }^{5}$. Ces auteurs soulignent qu'un niveau élevé de ce ratio indique un risque de défaut ou de crédit moindre. Par conséquent, en se basant sur cet argument, le signe prévu pour la variable ( $\triangle C A R)$ dans notre modèle (I.5) sera négatif en fonction du risque de crédit ( $\triangle$ CRRISK). La formule de calcul de ce ratio est :

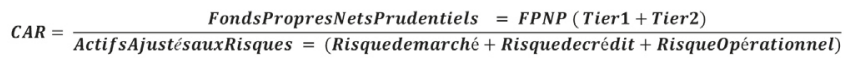

CRRISK : cette variable, qui exprime le niveau de prise de risque de crédit, est introduite dans notre modèle en tant que variable endogène à travers une variation première ( $\Delta$ CRRISK) entre $(t)$ et ( $t-1)$, et en tant que variable exogène en (t-1) pour refléter la principale source du risque bancaire. II s'agit d'un indicateur qui évalue le risque issu du bilan et du hors bilan à partir d'informations quantitatives relatives aux établissements et aux portefeuilles de crédits bancaires, à savoir le risque de contrepartie. II est mesuré par le rapport entre les provisions sur les créances douteuses et litigieuses, et le montant des concours accordés à l'économie selon la formule suivante :

$$
\text { CRRISK }=\frac{\text { Provisionssurlescréancesdouteusesetlitigieuses }}{\text { Concours à } l^{\prime} \text { économie }}
$$

En incorporant cette variable dans ses travaux, R. Aggarwal et K.T. Jacques (1998)soulignent qu'il s'agit d'une mesure qui

3. En plus des valeurs retardées de ces variables entre (t) et (t-1)

4. En Tunisie, la circulaire aux banques n 91-24 du 17 décembre 1991 définit quatre classes de risques (actifs classés). Classe1: actifs nécessitant un suivi particulier, Classe 2 : actifs incertains, Classe 3 : actifs préoccupants et Classe 4 : actifs compromis.

5. RWA : RiskWeightedAssets, et TA : Total Assets exprime la capacité de la banque à absorber les pertes liées à ses créances de mauvaise qualité. Elle reflète le niveau des actifs risqués dans un portefeuille d'actifs bancaires. Il en résulte que les banques, ayant plus de fonds propres, sont considérées comme moins risquées car elles constituent davantage de provisions sur leurs créances douteuses et litigieuses.

REG : introduite notamment par F. Heid et al. (2005), P. Van Roy (2005) et T. Jokipii et A. Milne (2010), cette variable exogène appréhende l'effet de la pression réglementaire sur les fonds propres, à travers des variables de contrôles composites, conformément aux directives des Accords de Bâle II et III, ainsi que des lois qui régissent les établissements de crédit en Tunisie. Plusieurs auteurs, comme P. Calem et R. Rob (1996), J.C. Godlewski (2005), ou encore F. Heid et al. (2006) ont souligné que l'effet de la réglementation bancaire sur le comportement des banques en matière de prise de risque aboutit à des résultats controversés. Pour une banque (i) à la fin d'une année (t), la variable pression réglementaire (REG) est définie de la manière suivante :

\section{REGi,t = [ Min REG + $\sigma$ CARi] - CAR i,t si CAR $i, t \leq$ Min REG $+\sigma$ CARi \\ REGi,t $=0$ si CAR $\mathbf{i}, \mathbf{t}>$ Min REG + $\sigma$ CARi}

Où Min REG est le minimum réglementaire exigé en matière de solvabilité, et oCAR l'écart-type du ratio de capital noté CAR.

BUFFER : cette variable a été introduite dans l'équation (I.5.1) de notre modèle pour expliquer la variation du niveau du ratio de capital réglementaire $(\triangle \mathrm{CAR})$ en fonction des fonds propres tampon, ou encore pour mesurer la distance prise par les banques par rapport au risque de non-conformité à la réglementation prudentielle des fonds propres. Cette variable, calculée à partir de la formule du ratio de couverture des risques, est définie comme la différence entre le ratio de capital (CAR) et le ratio minimum réglementaire exigé (Min REG). Pour la Tunisie, ce ratio était de $5 \%$ de 1990 à 2000, et de $8 \%$ sur la période 2001-2012. Pour F. Heid et al. (2004),il s'agit à la fois d'un stock de sécurité absolue de fonds propres détenus par la banque, mais également d'une mesure combinée de fonds propres et de risque de défaut. Cette variable est calculée selon la formule suivante :

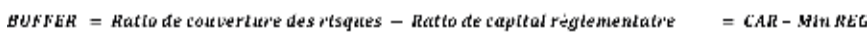

Nous avons choisi cette variable explicative car elle reflète le changement observé dans le montant de capital détenu, au-delà de celui exigé par le régulateur. Nous adoptons cette mesure du capital puisque nous supposons que les banques vont gérer leur fonds propres de manière à éviter ou réduire les coûts et pénalités associés à un non-respect des normes réglementaires. Cet argument a été notamment avancé par L. Maurin et M. Toivanen (2012).

Plusieurs auteurs ont introduit cette variable pour tenir compte du caractère pro-cyclique de l'environnement réglementaire ( $F$. Heid et al., 2004), ou encore pour détecter la relation entre le risque de crédit bancaire, la pression réglementaire et le comportement des ratios de solvabilité (M. Illing et G. Paulin, 2004). En particulier, les résultats des contributions de J. Ayuso et al. (2004) ont montré l'effet contra-cyclique des fonds propres tampon (Capital Buffer) pour des banques espagnoles. 
- (REG x $\triangle$ CAR)et (REG x $\Delta$ CRRISK) : sont deux variables composites qui expriment le produit de la variable reflétant la contrainte réglementaire par celle mesurant le niveau de fonds propres d'une part, et le produit du niveau du risque de crédit par la pression réglementaire d'autre part. Ces deux variables, inclues respectivement dans les équations (I.5.1) et (I.5.2) de notre modèle vont nous servir à quantifier l'impact de la réglementation sur le ratio de capital. En s'inspirant des travaux de T. Jokipii et A. Milne (2010), L. Maurin et M. Toivanen (2012) et S.L. Lin et al. (2013), nous pouvons supposer que la pression réglementaire affecte la vitesse d'ajustement du ratio de solvabilité des banques. Ce facteur d'interaction, défini comme le produit des deux variables, pourrait détecter de manière plus fine l'effet de la contrainte prudentielle sur le changement du niveau de capital de notre modèle. Le signe attendu de ces variables composites est positif pour le produit (REGx $\triangle \mathrm{CAR}$ ) par rapport à $\triangle \mathrm{CAR}$, mais, par contre, celui de $\left(\mathrm{REG}_{(\mathrm{i},-1-1)} \times \Delta \mathrm{CRRISK}(\mathrm{i}, \mathrm{t-1}) \mathrm{)}\right.$ semble imprévisible jusqu'à ce niveau d'analyse, comme l'indiquent D. Dahl et R. Shrieves (1990).

- (BUFFER x $\triangle$ CAR) et (BUFFER x CRRISK) : dans la même perspective, nous introduisons ces deux variables composites pour quantifier l'impact de la réserve des fonds propres sur les variations de niveau du risque de défaut. Ces mesures sont inspirées essentiellement des travaux de F. Heid, D. Porath et S. Stolz (2003) pour des banques allemandes, ainsi que de L. Maurin et M. Toivanen (2012) pour des banques européennes. À travers ces variables, nous examinons si les banques, quand elles possèdent des stocks de fonds propres tampon relativement faibles, ajustent leur niveau du risque de contrepartie plus rapidement et inversement.

\section{Méthodologie, échantillon et statistiques descriptives}

Cette section a pour objectif de décrire la stratégie de construction de notre base de données, et de présenter notre méthodologie ainsi que quelques statistiques descriptives sur notre échantillon.

\subsection{Constitution de l'échantillon et aspects méthodologiques}

Notre panel est constitué de dix banques commerciales tunisiennes sur une période de 23 années entre 1990 et 2012. Pour construire notre base de données, nous avons collecté les rapports annuels de ces établissements (bilan, compte de résultats, tableau des flux de trésorerie, engagements hors bilan et annexes), tous cotés à la $\mathrm{BVMT}^{6}$, ainsi que les rapports de Fitch-rating, I'APBEFT, la $\mathrm{BCT}$ et la $\mathrm{BAD}^{7}$. La liste des établissements retenus ainsi que

6. Bourse des Valeurs Mobilières de Tunis (BVMT).

7. Association Professionnelle des Banques et des Etablissements Financiers en Tunisie (APBEFT), Banque Centrale de Tunisie (BCT), Banque Africaine de Développement (BAD). les informations sur les ratios financiers les plus pertinents au 30 juin 2013 sont présentées dans le tableau 1. Le tableau 2 décrit les variables de notre modèle à deux équations simultanées que nous allons estimer par la méthode des triples moindres carrés (3SLS). Cette méthode a l'avantage d'avoir les mêmes propriétés que la méthode des moindres carrés ordinaires, mais sans biais et avec une variance minimale (W. Greene, 2006). Notre méthodologie consiste à commencer l'estimation de notre modèle en mettant l'accent sur les effets exercés par les variables explicatives, particulièrement la pression réglementaire et les fonds propres tampon, sur les variables endogènes ( $\triangle \mathrm{CAR}$ ) et ( $\triangle$ CRRISK).

Au cours de la période d'étude 1990-2012, nous avons distingué deux régimes d'exigence réglementaire en matière de capitalisation bancaire, ce qui nous a amenés à faire un découpage temporel en deux sous périodes; la première entre 1990 et 2000 lorsque le capital réglementaire minimum exigé était de plus de $5 \%$, et la deuxième de 2001 jusqu'à 2012 où le ratio de capital prudentiel devait être supérieur à $8 \%$.

\subsection{Propriétés statistiques des variables}

Nous constatons dans le tableau 1 ci-dessus que les ratios de capital réglementaire des dix banques retenues dans notre panel respectent la norme prudentielle en matière de solvabilité au 30 juin 2013. En effet, ces ratios varient entre $9.02 \%$ et $21.84 \%$ alors que le standard exigé est de $8 \%$. Par ailleurs, le ratio du risque de crédit, mesuré par la part des provisions sur les créances douteuses et litigieuses dans le montant des concours à l'économie, se caractérise par des valeurs qui expriment la mauvaise politique de provisionnement des banques tunisiennes. Cela s'explique en partie par la loi fiscale qui limite la déduction des provisions à un certain pourcentage des profits annuels ${ }^{8}$. En effet, ces taux de provisionnement, relativement faibles et jugés insuffisants ${ }^{9}$ compte tenu des garanties adossées à ces créances, ne respectent pas la norme prudentielle fixée à $70 \%{ }^{10}$. Les valeurs des fonds propres tampon (en \%) varient entre $1.02 \%$ et $13.84 \%$. Elles indiquent qu'en juin 2013, ces dix banques détiennent suffisamment de réserves en fonds propres (Capital Buffer). Elles montrent par ailleurs la capacité de ces établissements à éviter, ou encore à faire face, aux coûts et pénalités associés au non-respect potentiel des normes réglementaires en matière de solvabilité. Les probabilités de faillite calculées dans le tableau 1 indiquent que ces dix banques sont loin d'être sous

8. Par exemple $90 \%$ des crédits des banques commerciales sont destinés à financer le secteur privé, notamment le secteur des services (tourisme) et ceux affilés à la construction. Ainsi, les banques sont très exposées à des secteurs sensibles à un repli cyclique ou une récession.

9. Le taux de provisionnement est de l'ordre de $64,25 \%$ pour les banques privées, et à hauteur de $58,78 \%$ pour les banques publiques.

10. Les banques (en Tunisie) doivent constituer des provisions au moins égales à $20 \%$ pour les actifs de la classe 2, 50\% pour les actifs de la classe 3 et $100 \%$ pour lesactifs de la classe 4.Ces provisions doivent être affectées spécifiquement àtout actif classé égal ou supérieur à 50 mille dinars ou à $0,5 \%$ des fonds propres nets. 
Tableau 1. Liste des établissements retenus

\begin{tabular}{|c|c|c|c|c|c|}
\hline \multirow{2}{*}{ Banques } & \multirow{2}{*}{ Dénomination sociale } & \multicolumn{4}{|c|}{ Informations au 30/06/2013 } \\
\hline & & CAR & CRRISK & BUFFER & Prob. F \\
\hline ATTIJARI & Attijari Bank & $9,24 \%$ & $22,74 \%$ & $1,24 \%$ & $0,92 \%$ \\
\hline ATB & ArabTunisian Bank & $14,39 \%$ & $27,41 \%$ & $6,39 \%$ & $0,12 \%$ \\
\hline BIAT & Banque Internationale Arabe de Tunisie & $11,92 \%$ & $26,71 \%$ & $3,92 \%$ & $0,12 \%$ \\
\hline $\mathrm{BH}$ & Banque de l'Habitat & $10,23 \%$ & $19,11 \%$ & $2,23 \%$ & $0,14 \%$ \\
\hline BT & Banque de Tunisie & $21.84 \%$ & $53,37 \%$ & $13,84 \%$ & $0,11 \%$ \\
\hline STB & Société Tunisienne de Banque & $9.02 \%$ & $18,67 \%$ & $1,02 \%$ & $0,18 \%$ \\
\hline UBCI & Union Bancaire pour le Commerce et I'Industrie & $10.51 \%$ & 21,45 & $2,51 \%$ & $0,33 \%$ \\
\hline UIB & Union Internationale des Banques & $9.27 \%$ & $19,60 \%$ & $1,27 \%$ & $0,14 \%$ \\
\hline
\end{tabular}

Source: Calculs réalisés par les auteurs (Buffer et Prob F) suivant le rapport sur la supervision de la BCT 2013. CAR : ratio du capital réglementaire. CRRISK : ratio du risque de crédit. BUFFER : capitaux propres excédentaires ou "coussin de fonds propres", Prob. $F$ : Probabilité de faillite (Prob. $F=1 / 2 I R 2$ ) où IR est un indice du risque. IR $=[E(R O A)+C A R] / \sigma(R O A)$ avecE $(R O A)$ et $\sigma(R O A)$ qui sont respectivement la moyenne et l'écart type de la rentabilité des actifs (ROA.

Tableau 2. Description et spécification des variables

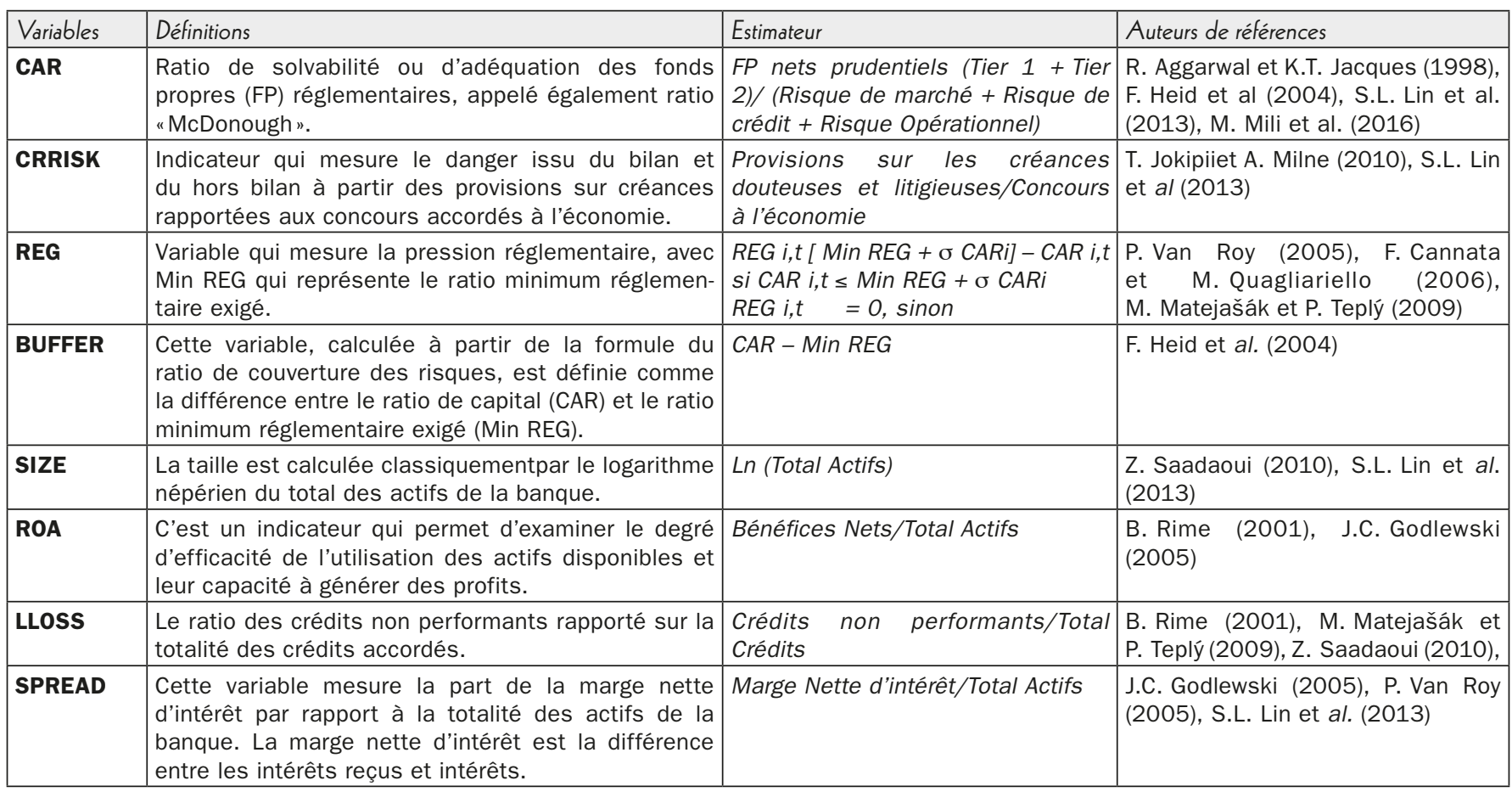

la menace d'une telle situation, car elles varient entre 0,11\% et $0.92 \%$. Généralement, un ratio de fonds propres plus élevé est associé à une probabilité de faillite plus faible. Cependant, J. Santos (2001) mentionne que le renforcement des exigences en capital peut inciter la banque à choisir des actifs plus risqués, ce qui réduit alors les effets positifs de cette augmentation et augmente finalement la probabilité de faillite. Ces auteurs insistent donc sur le fait que le renforcement des exigences en capital ne devrait donc pas se substituer à la surveillance et au contrôle du risque de crédit.

Les résultats du traitement statistique de notre échantillon dans le tableau 3 montrent que pour les 230 observations, le ratio du risque de crédit s'élève en moyenne à $18.28 \%$ avec une valeur maximale de $49.32 \%$; le ratio des fonds propres tampon affiche une moyenne négative de $-1.60 \%$ avec un écart type égal à $3.00 \%$. En général, sur ces 23 années, ces banques n'ont pas détenu suffisamment "de fonds propres". Cette situation s'explique par la faiblesse du ratio de solvabilité de ces banques, en majorité en dessous de la moyenne réglementaire exigée, surtout durant la première sous-période d'analyse.

De plus, la moyenne affichée sur les 23 années du montant du capital tampon (BUFFER) est négative (-1.60\%). Comme ces banques détiennent en fait des montants très volatiles de réserve de capital (écart type égal à $3 \%$ ), une amélioration de 
Tableau 3. Propriétés statistiques des variables retenues

\begin{tabular}{|c|c|c|c|c|c|}
\hline Variables & $(N)$ & Moyenne & Ecart-type & Min. & Max. \\
\hline$\triangle \mathrm{CAR}$ & 230 & 0.00351 & 0.01747 & -0.097418 & 0.070737 \\
\hline$\Delta$ CRRISK & 230 & 0.182811 & 0.108022 & 0.14941 & 0.493293 \\
\hline ROA & 230 & 0.005444 & 0.011517 & -0.103505 & 0.035006 \\
\hline SIZE & 230 & 14.81109 & 0.53435 & 12.4643 & 16.8847 \\
\hline SPREAD & 230 & 0.03631 & 0.021089 & 0.00935 & 0.12913 \\
\hline CAR (1) & 230 & 0.08256 & 0.03097 & -0.01098 & 0.17482 \\
\hline REG $x \Delta$ CRRISK (1) & 230 & -0.000451 & 0.002765 & -0.016535 & 0.015103 \\
\hline REG $\times \triangle C A R$ & 230 & 0.000970 & 0.000906 & -0.001293 & 0.002901 \\
\hline LLOSS & 230 & 0.227565 & 0.060468 & 0.12 & 0.32 \\
\hline CRRISK (1) & 230 & 0,58038 & 0,451900 & 0,000395 & 0,207632 \\
\hline REG (1) & 230 & 0.015944 & 0.018618 & 0 & 0.177708 \\
\hline
\end{tabular}

Nombre d'observations sur la période d'analyse (1990-2012). Min : valeur minimale observée. Max : Valeur maximale observée. Les variables ont été définies plus haut. (1) : Variable calculé en (t-1), $\Delta$ : signifie la variation de la variable entre (t) et (t-1).

leur niveau de solvabilité s'est opérée principalement (49\% en moyenne entre 2001 et 2012) par un amortissement du coussin de capital construit pour faire face au risque de crédit.

\section{Culture du risque et impacts globaux sur le ratio de capital et la prise de risque}

Avant d'estimer notre modèle (I.5), nous avons vérifié les conditions d'identification de nos deux équations simultanées (W. Greene, 2006),et l'absence du problème d'endogénéité pour les deux variables endogènes $\Delta \mathrm{CAR}_{\mathrm{i}, \mathrm{t}}$ et $\Delta \mathrm{CRRISK}_{\mathrm{i}, \mathrm{t}}$ par les tests de Durbin-Wu-Haussman, et de Davidson et Mckinnon. Le résumé des résultats de l'estimation par la technique 3SLS denotre modèle est exposé dans le tableau 4 suivant :

\subsection{Lien entre la variation du niveau de capital et le comportement de prise de risque de crédit}

Les hypothèses $(\mathrm{H} 1)$ et $(\mathrm{H} 2)$ précisent que la variation du niveau du ratio de capital de la banque est associée négativement au comportement de prise de risque de crédit des établissements bancaires, et réciproquement. Ainsi les paramètres $\left(\gamma_{4}\right)$ et $\left(\lambda_{4}\right)$ sont prévus avec un signe négatif dans les équations (I.5.1) et (I.5.2). Les résultats dans le tableau 4 nous permettent de constater que l'impact de la variation d'un changement du ratio de capital $\triangle C A R$ sur le comportement de la prise de risque de crédit $\triangle C R R I S K$ est négatif et statistiquement significatif au seuil de confiance de $90 \%$ pour l'intégralité de la période d'étude 1990-2012. L'effet est plus marqué sur la période 2001-2012 avec un risque statistique de seulement $1 \%$. Un changement moyen de $1 \%$ du niveau de solvabilité des banques de notre échantillon sur les 23 ans, se traduit en moyenne par une baisse importante de l'ordre de 98 pp du niveau du risque de crédit entre 1990-2012, contre 97 pp sur la période 2001-2012.

En revanche, chaque variation du niveau du risque de défaut de $1 \%$ se traduit par une variation inverse de $0.7 \%$ du niveau de ratio de capital pour la période 1990-2012. L'ajustement du niveau du risque par rapport au niveau de capital s'effectue de manière plus rapide et plus importante que l'effet inverse. Le comportement des banques en matière de prise du risque de contrepartie montre une association négative et statistiquement significative avec le changement du niveau de solvabilité avec un seuil de confiance de $10 \%$ pour la période globale et la souspériode 2001-2012. Ces résultats confirment donc nos deux hypothèses $\mathrm{H} 1$ et $\mathrm{H} 2$.

\subsection{Impact de la rentabilité des actifs sur la variation du ratio du capital}

Dans le tableau 4, les résultats de l'estimation montrent que pour les deux sous-périodes SP1 (1990-2000) et SP2 (2001-2012), ainsi que pour l'intégralité de la période d'étude, le ratio du rendement des actifs (ROA) est négativement et statistiquement significatif (au seuil de $5 \%, 1 \%$ et $10 \%$ respectivement) dans sa relation avec la variation du ratio de capital ( $\triangle \mathrm{CAR})$. En effet, le paramètre $\left(\lambda_{1}\right)$, prend des valeurs négatives $(-0.27742)$ sur SP1, et (-0.27780) sur SP2. Ce résultat contredit les résultats trouvés par F. Heid et al. (2003), M. Matejašák et P. Teplý (2009), Z. Saadaoui (2010), T. Jokipii et A. Milne (2010), et récemment S.L. Lin et al. (2013).

Nous concluons donc qu'en prenant en compte d'autres variables explicatives pour spécifier notre modèle, la relation entre ces composantes change de comportement. 
Tableau 4. Résultats de l'estimation du modèle (1.5)

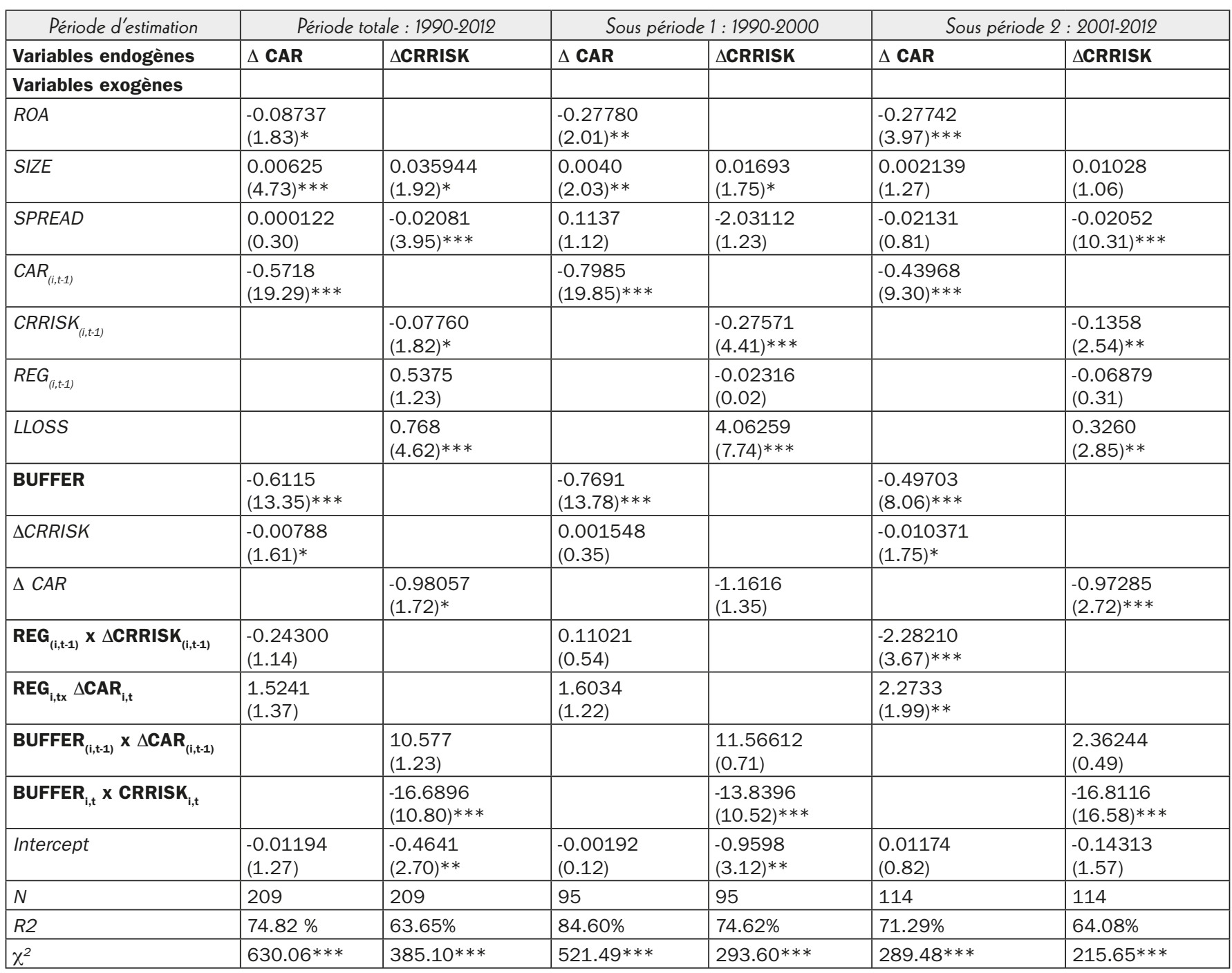

* : niveau de risque de $10 \%$, ** : niveau de risque de $\%$, *** : niveau de risque de $1 \%$. N : nombre d'observation. La statistique de Student figure entre parenthèses. $R^{2}$ : coefficient de détermination. $\chi^{2}$ : statistique de chi-deux (test de Wald) selon la méthode $3 S L S$.

La majorité des théoriciens ont conclu que la qualité des portefeuilles d'actifs, dont disposent les banques, est supposée varier dans le même sens que la variation du ratio de solvabilité réglementaire car une fraction des fonds propres est constituée par des bénéfices reportés (A. Guidara et al., 2013).

En effet, les banques, qui enregistrent des profits importants au cours d'un exercice (t-1), disposent ensuite de résultats à reporter, ce qui se traduit par un niveau important des fonds propres ou du ratio de capital au début de l'exercice suivant (t) comme le souligne L. Maurin et M. Toivanen (2012). Mais B. Rime (2001) a mis en évidence, qu'en cas d'asymétrie d'informations sur le marché, les signaux, émis par la divulgation de l'information sur le bénéfice influent sur le sens de cette relation qui devient alors négative.

Finalement, cette relation inverse peut s'expliquer par le fait que l'amélioration de la qualité du portefeuille d'actifs des banques de notre échantillon se fait au détriment de leur solvabilité; car elles distribuent majoritairement leurs bénéfices aux actionnaires, au lieu de les affecter à l'accroissement de leur niveau de capital. Elles utilisent donc d'autres moyens pour financer cette augmentation. En effet, une amélioration du ratio (ROA) de $1 \%$ entraine une baisse de $22.77 \%$ de son niveau de ratio de capital.

\subsection{Impact de l'effet taille}

Si la taille d'une banque augmente, alors cette dernière devraitavoir tendance à être plus solide et prendre moins de risque (notamment à cause de la diversification de ses activités).

L'effet "taille" sur les variables ( $\triangle$ CAR) et ( $\triangle$ CRRISK) dans le tableau 4, témoigne de l'existence d'un lien positif et statistiquement significatif (avec un risque de $1 \%$ et de $10 \%$ respectivement) durant la période 1990-2012 pour les dix banques universelles, et pour la sous-période SP1. Le fait que ces deux indicateurs ne 
sont pas significatifs pour SP2 pourrait indiquer un changement de la relation sur la période 2001-2012.

Ce résultat est contraire à la plupart des travaux empiriques antérieurs. En particulier, D. Dahl et R. Shrieves (1990), J.C. Godlewski (2005) etZ. Saadaoui (2010) ont trouvé, quant à eux, un effet taille négatif sur le niveau du ratio de capital. Par ailleurs, T. Jokipii et A. Milne (2010) et S.L. Lin et al. (2013) ont détecté un impact significatif et négatif de la taille sur le niveau de risque ( $\triangle \mathrm{CRRISK}$ ). Toutefois, ces résultats sont à temporiser suivant les pays car D. Dahl et R. Shrieves (1990) rappellent que les opportunités d'investissement de la banque, les caractéristiques de propriétés et l'accès facile aux marchés interbancaires pour se refinancer pouvait influencer ces relations.

Comme nous l'avons déjà mentionné, les banques tunisiennes sont toutes de tailles homogènes et qualifiées de petite taille, contrairement aux banques européennes ou encore américaines. De même, le volume des fonds propres détenu par ces dix banques est aussi modeste par rapport à celui détenu par les banques étrangères. En fait, ces dix banques tunisiennes n'arrivent pas à baisser leurs niveaux de prise de risque de crédit, même si leur taille s'améliore. Cela peut s'expliquer par le manque de diversification qui caractérise leur activité. Ce résultat contredit ceux de R. Aggarwal et K.T. Jacques (1998)et F. Heid et al. (2004), mais confirment ceux trouvés de A. Awdeh et al (2011) et A. Guidara et al. (2013). Toutefois, ce résultat empirique n'est plus valide sur la dernière période de 2001-2012, et semble indiquer une évolution de l'effet taille sur la gestion des risques en Tunisie. Une croissance de la taille des grandes banques tunisiennes ne les inciterait plus à prendre plus de risque cette dernière décennie.

\subsection{Impact du «Capital Buffer» sur la variation du ratio de fonds propres}

L'impact des fonds propres tampon sur le niveau de ratio de capital est négatif et statistiquement significatif. En effet, les résultats de l'estimation confirment notre troisième hypothèse. Le signe du paramètre $\left(\lambda_{6}\right)$ est négatif, comme prévu pour la période globale de 1990 à 2012 pour l'ensemble des dix banques de notre panel, aussi que pour les deux autres sous-périodes. Les coefficients d'ajustement du capital buffer par rapport au niveau de capital s'élèvent à (-0.6115), (-0.7691) et à (-0.49703) avec des T-Student de (13.35), (13.78) et (8.06) respectivement, et sont statistiquement significatifs avec un risque de $1 \%$ sur les trois périodes d'analyse.

En moyenne, chaque augmentation du niveau du stock de fonds propres (Capital Buffer) se traduit par une baisse du niveau du ratio de capital de l'ordre de $61 \%$ sur toute la période pour les dix établissements. Ce type de réserve présente un caractère contra-cyclique qui peut s'expliquer par le mouvement cyclique dans les exigences des fonds propres. En effet, la hausse du niveau du capital d'une banque, en cas de bonne conjoncture et prolifération de distribution des crédits, se traduit par une diminution du stock de réserve en fonds propres, lequel vise à absorber les pertes inattendues et par conséquent à réduire le risque d'insolvabilité. Cette relation est qualifiée de normale car pour s'aligner sur la norme réglementaire du ratio de solvabilité requis, les fonds propres exigés augmentent lorsque la conjoncture économique s'avère bonne et le "coussin des fonds propres" s'épuise lorsque la situation financière se dégrade (M. Illing et G. Paulin, 2004). Les autorités réglementaires s'inquiètent d'une telle cyclicité du capital car elle peut être de nature à bouleverser la stabilité du système financier et bancaire.

Nos résultats corroborent ceux trouvés par J. Ayuso et al. (2004) qui ont détecté une association négative entre le cousin de capital et le cycle économique, qui se traduit par une variation du niveau des fonds propres de la banque plus importante en cas de récession qu'en cas d'expansion. Ces auteurs ont remarqué aussi que ce "coussin" est susceptible de réduire la volatilité du capital, résultat qui pourrait s'expliquer par le fait que certaines banques gardent un niveau de capital supplémentaire en cas d'expansion pour l'utiliser lors de récession. Ces constatations sur la réaction à la hausse du coussin de capital en cas de récession due à la réduction du niveau des activités de crédits (baisse du niveau du capital) ont été confirmées par A. Guidara et al (2013). Ces auteurs ont repéré que sous pression réglementaire, le coussin de capital diminue en cas de récession financière et par conséquent, compense la réduction des prêts accordés.

\section{Impacts spécifiques des prêts non performants, du ratio de marge et de la réglementation}

Après avoir montré les impacts globaux, nous allons dans cette section analyser plus en profondeur, les impacts des prêts non performants, du ratio de marge et de la pression réglementaire sur le comportement de prise de risque de crédit des dix banques de notre échantillon.

\subsection{Culture du risque et impact des prêts non performants sur le comportement de prise de risque de crédit}

Le tableau 4 montre qu'il existe une relation positive et statistiquement significative entre le ratio des prêts non performants (LLOSS) et la prise de risque de crédit ( $\triangle$ CRRISK) des dix banques sur les trois périodes de notre estimation (avec respectivement un risque de $1 \%$ pour la période totale et SP1, et de $5 \%$ pour SP2). Cette association positive a été notamment mentionnée par B. Rime (2001), P. Van Roy (2005), Z. Saadaoui (2010), A. Awdeh et al. (2011), L. Maurin et M. Toivanen (2012) et S.L. Lin et al. (2013) qui associent l'accroissement des prêts non performants à celui du risque global de la banque, appréhendé par le risque de défaut lequel menace le plus l'activité et la profession bancaire. 
Nos résultats corroborent également ceux trouvés par F. Cannata et M. Quagliariello (2006) et de R. Aggarwal et K.T. Jacques (2001), à savoir que les banques détenant des prêts de mauvaise qualité s'engagent dans des activités plus risquées.

\subsection{Lien entre le ratio de marge nette d'intérêt, la variation du ratio de capital et la prise de risque de crédit}

Les résultats de l'estimation 3SLS montrent un impact négatif et significatif de la marge nette d'intérêt bancaire (SPREAD) sur la prise de risque de crédit pendant toute la période d'étude (1990-2012) et pour SP2 (2001-2012).

Par contre, la variable SPREAD n'a pas d'effet statistiquement significatif sur le niveau du ratio de capital pour les trois périodes d'analyse. Ainsi, le niveau de solvabilité de ces banques ne peut pas être expliqué par les revenus provenant de sa fonction d'intermédiation en termes d'intérêts. Cette absence d'effet montre que l'amélioration de la marge d'intérêt bancaire ne se traduit pas forcément par une augmentation du niveau de capital comme l'ont déjà souligné J.C. Godlewski (2005), P. Van Roy (2005)et S.L. Lin et al. (2013) dans d'autres contextes.

En revanche, notre résultat concernant l'effet inverse de cette marge sur l'incitation à la prise de risque de crédit, a également été soutenu par les travaux de Z. Saadaoui (2010), A. Awdeh et al (2011) et L. Maurin et M. Toivanen (2012). Ces auteurs stipulent que lorsque les banques distribuent davantage de crédits, le volume d'intérêts reçus en parallèle tend à augmenter, mais pas forcément la marge d'intérêt à cause des défauts. En effet, en cas de défaut de l'emprunteur, la marge d'intermédiation se dégrade même si les ressources collectées sous formes de dépôts ne sont pas rémunérés. L'une des techniques possibles, pour absorber l'accroissement du niveau du risque de crédit, est la révision des taux et des garanties des emprunts octroyés à des clients jugés peu solvables dans l'objectif de mieux couvrir les risques.

\subsection{Relation entre les variables composites, la variation du niveau de capital et l'incitation à la prise de risque de crédit}

Les résultats de l'effet des deux variables relatives à la pression règlementaire à savoir $\left(\mathrm{REG}_{(\mathrm{i}, \mathrm{t}-1)} \times \Delta \mathrm{CRRISK}(\mathrm{i,t-1)})\right.$ et $\left(\mathrm{REG}_{\mathrm{i}, \mathrm{t}} \times \Delta \mathrm{CAR}_{\mathrm{i}, \mathrm{t}}\right)$ sur la variation du niveau de ratio de capital ( $\triangle \mathrm{CAR}$ ) confirment qu'elles ont un impact statistiquement significatif sur la capitalisation des dix établissements bancaires uniquement sur la période SP2 (2001-2012).

La pression réglementaire conjuguée au comportement de prise de risque de crédit ex-ante soit $\left(\mathrm{REG}_{(\mathrm{i}, \mathrm{t}-1)} \times \Delta \mathrm{CRRISK}_{(\mathrm{i}, \mathrm{t}-1)}\right)$ a un impact négatif sur le niveau de solvabilité $\left(\lambda_{7}=2.28210\right)$ avec un seuil de confiance de $99 \%$. De plus, la variable composite $\left(R E G_{i, t} \times \Delta C A R_{i, t}\right)$ a un impact positif sur le niveau de solvabilité des banques tunisiennes. Le paramètre $\left(\lambda_{8}\right)$ est positif (2.2733) avec un p-value de (1.97),et est statistiquement significatif au seuil de risque de $5 \%$. Ces résultats confirment ceux de R. Jacques et K.T. Aggarwal (1998), L. Maurin et M. Toivanen (2012) et S.L. Lin et al (2013). La réaction de ces établissements envers les exigences réglementaires se manifeste par la baisse du niveau de l'incitation à la prise de risque de crédit sur la SP2. C'était justement l'effet recherché par le durcissement de la réglementation prudentielle en Tunisie, comme dans d'autres pays (N. Janson et J-M. Sahut, 2010). En revanche, la variable pression réglementaire seule $\left(\mathrm{REG}_{(\mathrm{t}-1)}\right)$ n'a aucun impact sur le niveau de la prise de risque de crédit sur les trois périodes de notre analyse. Ce résultat corrobore celui trouvé précédemment. En effet, une fois que les ratios de solvabilité des banques sont conformes à la norme de gestion prudentielle exigée, la réglementions n'a plus d'effet (T. Jokipiiet A. Milne, 2010).

Concernant la variable composite (BUFFER x CRRISK), les résultats de l'estimation de notre panel montrent qu'elle est associée négativement avecun seuil de confiance de $99 \%$ sur les trois périodes. En particulier, le paramètre $\left(\gamma_{8}\right)$ prend une valeur négative (-16.6896) entre 1990 et 2012 avec un T-Student d'environ (10.80).

Ainsi, une hausse des fonds propres tampon (associés au risque de crédit) pousse les banques à ajuster leurs incitations à la prise de risque. Ce résultat réaffirme les évidences trouvées par F. Heid et al. (2004), qui a traité des déterminants du coussin de capital et en particulier du risque de contrepartie. Ses principaux résultats indiquent une relation négative entre le coussin de capital et le risque de défaut pour ces firmes. Ils supportent I'hypothèse selon laquelle les fonds propres tampon sont considérés comme une couverture contre les coûts associés aux mesures disciplinaires réglementaires. Par contre, la variable composite (BUFFER x CAR) n'est pas significative.

\section{Conclusion}

L'objectif principal de cet article était d'analyser la gestion et la culture du risque de crédit dans le cadre du renforcement de la réglementation bancaire dans un pays émergent, la Tunisie. Cet article quantifie l'impact simultané des variations du ratio de capital sur le comportement de prise de risque de défaut par le management, sous la pression réglementaire. Nous avons estimé un modèle à deux équations, qui englobe plusieurs variables caractérisant le capital et le risque de crédit bancaire, développé initialement par D. Dahl et R. Shrieves (1990)et amélioré récemment par S.L. Lin et al. (2013). Nous avons sélectionné un échantillon des dix plus importantes banques tunisiennes sur une période de 23 années allant de 1990 jusqu'à 2012, divisée par la suite en deux sous-périodes compte tenu des changements réglementaires intervenus. 
Les estimations de notre modèle par la technique des 3SLS ont permis de dégager trois résultats significatifs ayant des implications managériales :

- (1)les banques tunisiennes ont amélioré leur solvabilité sur la période 2011-2012, avec le durcissement des contraintes réglementaires sur l'exigence des fonds propres,

- (2) les contraintes réglementaires sur l'exigence des fonds propres n'ont aucune incidence sur le comportement de prise de risque de crédit des banques, maiscette pression institutionnelle est associée négativement au ratio de couverture des risques essentiellement sur la période 2001-2012,

- (3)le management des banques révèle que le "stock de réserves" de capital (Capital Buffer) est lié négativement au niveau du ratio de capital de ces banques.

Finalement, l'effet simultané entre la variation du niveau du capital et l'incitation à la prise de risque de crédit par le management de ces banques, s'avère négatif et statistiquement significatif sur toute la période d'analyse. Le changement du niveau des fonds propres se traduit par une répercussion importante et rapide sur l'incitation du management à la prise de risque de contrepartie et sur le niveau de provisionnement (culture du risque conservative) car ces banques modulent leurs ratios de capital surtout en fonction de l'ampleur du risque d'insolvabilité de leurs clientèles.

Nous avons mis l'accent aussi sur la gestion du risque de crédit bancaire et le comportement des banques dans le développement de la culture du risque. Les conclusions obtenues nécessitent de poursuivre cette recherche afin de détecter également l'impact du niveau du risque de liquidités sur le comportement du ratio de capital sous la pression réglementaire.

\section{Bibliographie}

Aggarwal R. \& Jacques K.T. (2001), “The impact of FDICIA and prompt corrective action on bank capital and risk : Estimates using simultaneous equations model", Journal of Banking and Finance, vol.25, pp. 1139-1160.

Aggarwal R. \& Jacques, K.T. (1998), "Assessing the impact of prompt corrective action on bank capital and risk", Economic Policy Review, vol.4, n 3, pp. 23-32. Altman E.I. (1968), "Financial ratios, discriminant analysis and the prediction of corporate bankruptcy", The Journal of Finance, vol. 23, n 4, pp. 589-609. Arouri M., Boubaker S. \& Nguyen D.K. (2013), Emerging Markets and the Global Economy : A Handbook, Elsevier, Academic Press, 928 p.

Awdeh A., El-Moussawi C. \& Machrouh F. (2011), “The Effect of Capital Requirements on Banking Risk", International Research Journal of Finance and Economics, ${ }^{\circ}$ 66, pp. 133-146.

Ayuso J., Perez D. \& Saurina J. (2004), “Are capital buffers pro-cyclical? Evidence from Spanish panel data”, Journal of Financial Intermediation, $\mathrm{n}^{\circ} 13$, pp. 249-264.

Bellalah M. (2006), "Liens Entre les Fonds Propres Bancaires, La Réglementation Prudentielle et le Risque de Crédit Dans le Contexte Tunisien : Une Approche en Termes de Causalité ", Euro MediterraneanEconomics And Finance Review, vol. 1, $n^{\circ} 3$, June.

Calem P. \& Rob R. (1999), “The impact of capital-based regulation on bank risk-taking", Journal of Financial Intermediation, n 8, pp. 317-352.

Cannata F. \& Quagliariello M. (2006), “Capital and risk in Italian banks : A simultaneous equation approach", Journal of Banking Regulation, vol.7, pp. 283-297.

Chiuri M.C., Ferri G. \& Majnoni G. (2002), “The macroeconomic impact of bank capital requirements in emerging economies: Past evidence to assess the future", Journal of Banking and Finance, vol.26, pp. 881-904.
Dahl D. \& Shrieves R. (1990), 'The Impact of Regulation on Bank Equity Infusions', Journal of Banking and Finance, vol.14, n 6, pp. 1209-1228.

Demsetz R.S. \& Strahan P.E. (1997), “Diversification, size, and risk at U.S. bank holding companies", Journal of Money, Credit, and Banking, vol. 29, n³, pp. 300-313.

Ediz T., Michael I. \& Perraudin W. (1998), “The impact of capital requirements on U.K. bank behaviour", Economic Policy Review, Federal Reserve Bank of New York, October, pp. 15-22.

Godlewski J.C. (2005), "Bank capital and credit risk taking in emerging market economies”, Journal of Banking Regulation, vol.6, n², pp. 128-145.

Greene W. (2006), Econométrie, Pearson Education, France, 946 p.

Gropp R. \& Heider F. (2009), “The Determinants of Bank Capital Structure”, European Central Bank Working Paper Series, n 1096,pp. 1-50, September. Guidara A., Van Son L., Soumaré I. \& Tchana F.T. (2013), “Banks' Capital Buffers, Risk and Performance in the Canadian Banking System : Impact of Business Cycles and Regulatory Changes", Journal of Banking and Finance, vol. 37, n 9, pp. 3373-3387.

Heid F., Porath D. \& Stolz S. (2003), “Does capital regulation matter for bank behavior? Evidence for German savings banks", Working Papers, Kiel Institute for World Economics, $n^{\circ} 1192$, Deutsche Bundesbank.

Illing M. \& Paulin G., 2004, "The New Basel Capital Accord and the Cyclical Behaviour of Bank Capital," Staff Working Papers 04-30, Bank of Canada.

Jacques K.T., Nigro P. (1997), 'Risk-based capital, portfolio risk and bank capital : A simultaneous equations approach', Journal of Economics and Business, vol.49, pp. 533-547.

Janson N. et Sahut J-M. (2010), "Stabilité du secteur bancaire : Des tests réellement utiles?", Banque \& Stratégie $n^{\circ} 282$.

Jokipii T. \& Milne A. (2010), "Bank capital buffer and risk adjustment decisions", Journal of Financial Stability, vol.7, $\mathrm{n}^{\circ} 3$, pp. 165-178.

Kleff V. \& Weber M. (2008), “How Do Banks Determine Capital ?Evidence from Germany", German Economic Review, vol.9, n 8, pp. 354-372.

Lev B. (1969), “Industry Averages as Targets for Financial Ratios," Journal of Accounting Research, autumn, pp. 290-299.

Lin S. L, Hwang D. Y, Wang K.L. \& Xie Z.W. (2013), "Banking Capital and Risk-taking Adjustment under Capital Regulation: The Role of Financial Freedom, Concentration and Governance Control", International Journal of Management, Economics and Social Sciences, vol.2, pp. 99-128.

Lintner J. (1977), “Discussion” In Financial Crises : Institutions and Markets in a Fragile Environment, Ed. E.I. Altman and A.W. Sametz, pp. 204-207. New York : John Wiley and Sons.

Massiera B. (2007), "Culture d'entreprise, l'échec d'un concept", Communication, vol.25, $\mathrm{n}^{\circ} 2, \mathrm{pp}$ 131-147.

Matejašák M. \& Teplý P. (2009), “Regulation of bank capital and behavior of banks : Assessing the US and the EU-15 region banks in the 2000-2005 period", IES Working Paper, 23/2009, IES FSV, Charles University, Prague, République tchèque.

Maurin L. \& Toivanen M., (2012), “Risk, capital buffer, and bank lending : A granular approach to the adjustment of euro area banks", ECB Working Paper, $\mathrm{n}^{\circ} 1499$.

Mili M., Sahut J-M, Trimeche H. \& Teulon F. (2016), "Determinants of the Capital Adequacy Ratio of Foreign Bank's Subsidiaries : The Role of Interbank Market and Regulation". Research in International Business and Finance, février.

Ospital D. (2006), "Le risque opérationnel, ou l'opportunité unique pour les banques de s'approprier une véritable culture du risque", Revue d'économie financière, vol.84, $\mathrm{n}^{\circ} 3$, pp. 105-119.

Powell A. (2004), "Basel II and developing countries : sailing through the sea of standards", Policy Research Working Paper, World Bank, n³387, September. Rime B. (2001), "Capital requirements and bank behavior : Empirical evidence for Switzerland”, Journal of Banking and Finance, vol.25, n 4, pp. 789-805. Saadaoui Z. (2010), "Capitalisation et prise de risque des banques dans les pays émergents", Brussels Economic Review - cahiers économiques de Bruxelles, vol.53, n³/4, Autumn-Winter.

Sahut J-M et Mili M. (2009), "Quelques mythes et réalités sur la crise des subprimes", Revue Gestion 2000, vol.26, n 6, pp. 19-33.

Santos J. (2001), 'Bank capital regulation in contemporary banking theory: A review of the literature', Financial Markets, Institutions and Instruments, vol.10, pp. 41-84.

Van Roy P. (2005), “The impact of the 1988 Basel Accord on bank capital ratios and credit risk-taking: an international study", Finance 0509013. Economics Working Paper, Archive EconWPA. 